\title{
Creating and running worldwide accident scenarios with JRodos
}

\author{
C. Landman ${ }^{1}$, W. Raskob ${ }^{1}$, D. Trybushnyi ${ }^{1}$ and I. Ievdin ${ }^{2}$ \\ 1 Karlsruhe Institute of Technology (KIT), Postfach 3640, 76021 Karlsruhe, Germany. \\ 2 Ukrainian Center for Environmental and Water Projects, prosp. Glushkova 42, 03187 Kiev, Ukraine.
}

\begin{abstract}
The article summarises ingredients and constraints for the development of a nuclear accident scenario, and describes the features and tools of the decision support system JRodos that are useful to assist the developer in creating and running such scenarios.
\end{abstract}

Keywords: JRodos worldwide / nuclear accident scenario creation

\section{Introduction}

In the past few years, the decision support system JRodos (Ievdin et al., 2010) has been used to generate several comprehensive scenarios for nuclear accidents in Europe, for case studies as well as for demonstrations and training. Among others, longer-duration scenarios were developed for the First NERIS Platform Workshop, February 2012, in Bratislava and the NERIS-TP Training Course on Preparedness for Nuclear and Radiological Emergency Response and Recovery, October 2013, in Trnava. In 2013 the JRodos worldwide facility became available, which extended the range of application for such purposes to any position on the globe. Based on this experience, the ingredients and constraints for a nuclear accident scenario development are summarized, and the features and tools of JRodos that are useful to assist the developer in creating and running such scenarios are described.

\section{Ingredients and constraints for a nuclear accident scenario development}

Case studies, demonstrations or training sessions for radioecological or radioprotection purposes require expressive accident scenarios to illustrate the significant points of the given sets of issues. With respect to the preparedness for nuclear or radiological emergencies with the potential for significant off-site radiological and societal consequences, categorized accident scenarios are needed to support the preparation of countermeasure strategies in the different accident phases.

In any case, such scenarios consist of the following basic ingredients:

- information about the release: the source term is the prime determinant for the possible range of accident consequences - one that is "too weak" cannot lead to significant consequences, and one that is "too strong" may lead to unacceptable consequences for the scenario purpose, even with the most favorable weather for the given goal;

- a nuclear power plant or a release location for a radiological emergency where the assumed event takes place: if it does not necessarily need to represent a real-world facility, this gives a further degree of freedom when tuning the scenario;

- a date when the assumed event takes place: whether the accident date lies within the growing season or outside plays a crucial role for food-chain model assessments;

- information about the meteorological conditions during the assumed dispersion and deposition phase for airborne releases, and about hydrological and possibly also meteorological conditions in the considered dispersion time interval for aquatic releases;

- geo-referenced data for the area and locations under consideration that are required for the operation of the model(s) to be used;

- additional details about the region and specific locations if required for special applications, and

- maps that cover the area and specific locations under consideration for displaying the accident scenario background, the radiological situation and model results.

When creating a case study, demonstration or training scenario, the room for development is largely determined by externally given conditions concerning the degree of realism for the release scenario and the weather and map-related specifications on the one hand, and on the other hand, by the requirements of the purposes the scenario will serve. It may well turn out that it is simply impossible to achieve a given scenario goal by dutifully obeying all constraints. For example, a prescribed realistic source term in connection with prescribed recorded weather data and a real release location with real respective maps fails to achieve a contamination pattern that would be necessary to illustrate recovery phase techniques in 
urban areas. In such a case, the scenario supplier would negotiate with the client for the scenario to weaken the constraints, or to accept some deliberate violation of a constraint.

Accident scenarios for preparedness considerations and for probabilistic accident consequence studies, e.g. for licensing of nuclear power plants, combine categorized source terms with a large number of weather sequences that are selected from data sets covering one year or more under given statistical assumptions. Running applications of this type was previously the domain of computer codes such as COSYMA (KfK and NRPB, 1991) in the European Union and MACCS (Chanin et al., 1990) in the US, that generated the complete result spectrum and performed the probabilistic analysis within one run. Nowadays, the development in information technology also allows tackling these problems with modern decision support systems such as JRodos, separating the result generation from the statistical analysis that will follow.

\section{General features of JRodos that assist in developing and running scenarios}

Generating training or demonstration scenarios often needs considerable fine-tuning to achieve a suitable radiological situation, in particular when operating under externally given constraints for the scenario development, and frequently involves a larger number of calculations with slightly different initial assumptions, in particular about the source term and weather conditions. Such a task is significantly facilitated by the possibility in JRodos to save the complete user input as an xml file, and then to reuse the input - possibly after some modification for a new calculation. This procedure can be repeated interactively, calculation for calculation. In another approach, a number of xml files is generated interactively, but the respective calculations are performed in a batch-job-like manner without user interaction by placing a bulk of the $\mathrm{xml}$ files in a certain directory. From there they are automatically picked up by the system, the calculations are carried out and the generated results are saved; this feature is particularly attractive when the calculation times become bothersome. The latter method was applied recently for a case study in Germany (Gering et al., 2013).

With respect to studies that require the analysis of a large number of weather sequences with otherwise identical conditions, the method will be extended to enable a separate and automated processing with statistics tools by attributing in a formalized way each set of results to the generating meteorological sequence.

In JRodos, the navigation to model results is performed via browsing through a "Result Tree" which represents the individual calculation results as "leaves" in folders and subfolders. The total number of available results can be huge, depending on the generating physical model. In this context, another useful feature is the possibility to pre-select the results that appear in the Result Tree, thus clipping the amount of results to exactly those complying with the application demands of the scenario. Such a pre-selection can be saved and reused and also be made available for other users.

A "map view" visible in the JRodos Main Window Tab consists of a combined display of one or more layers of geo-referenced information, including map-type calculation results. On delivery, the system contains a default set of nonresult layers covering most of Europe. Additional layers can be loaded as files: currently supported are the two common geographical data formats "ESRI Shapefile" (*.shp) and "Tagged Image File Format" (*.tiff, *.tif). Layers can also be loaded from a PostGIS database (JRodos internal or external), or from a Web Map Service (WMS) server. It is also possible to use Google Maps (hybrid view) or OpenStreetMaps as background layers; in particular, the latter are helpful when better maps are not available or the map view will be used for a publication. For the preparation of scenario documents and accompanying presentations, the system's possibility to save a geo-referenced result representation ("map") as a static picture - or for time-dependent results also as animated gif files - is an indispensable feature.

Because the user input, the result selection, and selected map views including result examples can be kept on a storage medium, it is possible to distribute complete scenarios and application examples to course participants or other interested users who can utilize them on their own, provided that all other data required for running the models - such as recorded weather data files - are also supplied. Recently, a library has been made available that collects complete scenarios for general use in the JRodos community ${ }^{1}$.

The JRodos Results Guide facilitates finding the required results in the JRodos Result Tree, at least for the results from the models that make up the JRodos EMERGENCY model chain:

- the Local-Scale Model Chain (LSMC; a near-range atmospheric dispersion model coupled to a meteorological preprocessor);

- the model for simulation of early countermeasures and doses in the near range (EmerSim);

- the model for deposition calculations for FDMT (DepoM), and

- the food chain and dose model, terrestrial (FDMT).

For each physical result class, a detailed and precise definition is given. In addition, the results are classified with respect to their possible application. The identified classes cover results allowing at-a-glance identification of the severity of the situation; results relevant for the analysis of the development of the radiological situation until the end of the calculation episode; results useful for the evaluation of early countermeasures with respect to extent, feasibility, efficiency and sensitivity to uncertainties; and results showing the longer-term development of the radiological situation.

\section{JRodos worldwide application}

Atmospheric dispersion and deposition and other model calculations require knowledge about:

- characteristics of the nuclear facility causing the event, such as co-ordinates of the release location;

\footnotetext{
1 jrodos@ resy5.fzk.de:/JRodosTraining/Scenarios.
} 


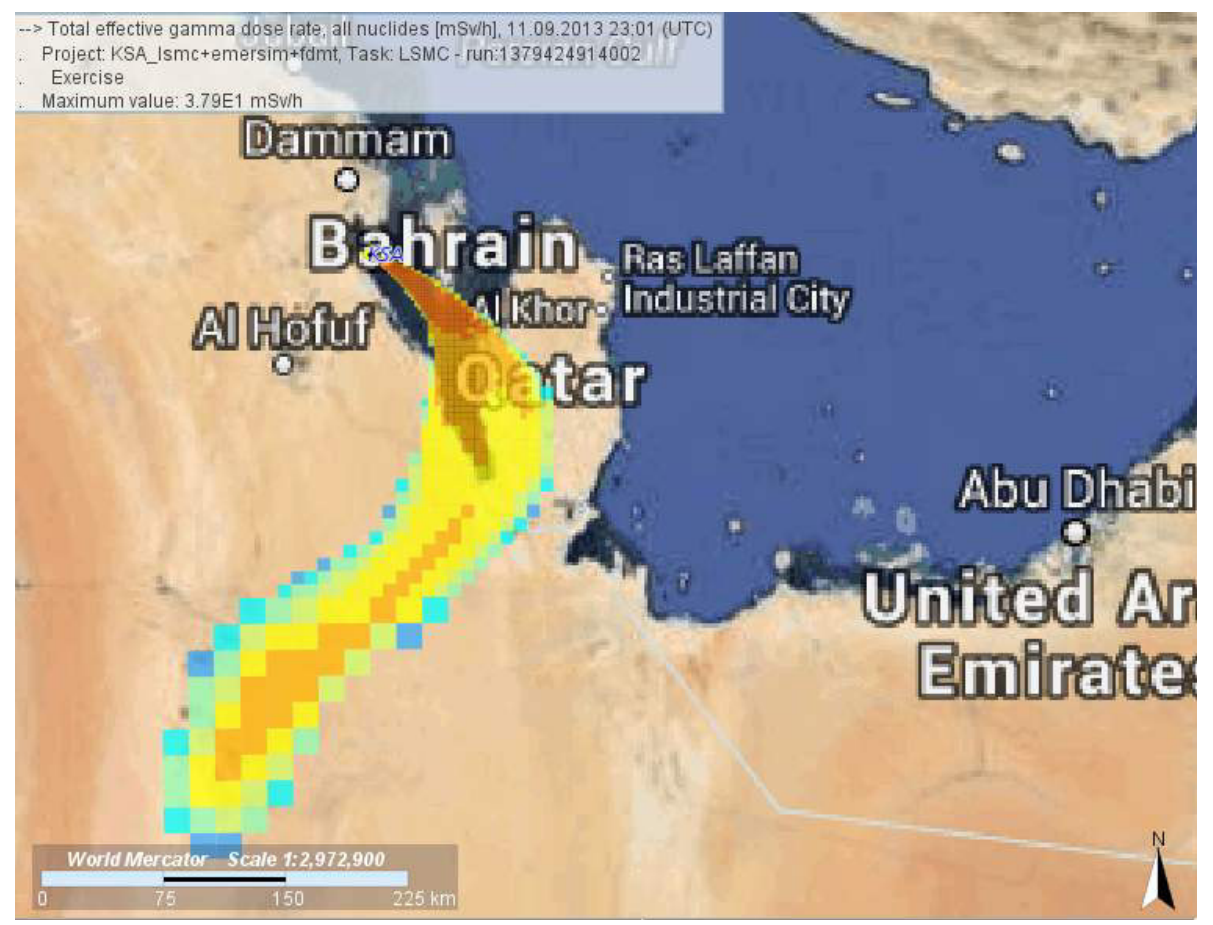

Figure 1. Total effective gamma dose rate, using NOMADS weather data (JRodos screenshot).

- geographical characteristics for the surroundings of the release location (elevation, land use, soil type, population density and agricultural production - also called "engine" data), and

- the atmospheric conditions determining the dispersion and deposition of the released radioactive material.

With respect to the first two categories, JRodos first contained default information for all European nuclear power plants and engine data covering most parts of Europe. To allow use of JRodos outside the range of the default data, worldwide applicable data was collected from public sources and included in the standard delivery package.

If the scenario development requires the use of real-world meteorological data instead of made-up weather information by the user, data from national weather services should be employed whenever available. If this is not the case, an interface to the freely available and globally applicable numerical weather forecast and reanalysis data from the NOMADS (NOAA Operational Model Archive and Distribution System) service in the $\mathrm{USA}^{2}$ is now applicable in JRodos, which allows downloading and applying such data for a user-specified time period. The NOMADS data are available in two formats: Grib1 with 1 degree $<=>100 \mathrm{~km}$ pixel size, and Grib2 with 0.5 degree $<=>50 \mathrm{~km}$ pixel size. The data covers a forecast period of seven days, appearing every 6 hours for 3-hour time steps. One such set covers the whole world.

The global NOMADS data has a relatively coarse spatial resolution and does not take account of conditions that influ-

2 The US National Oceanic and Atmospheric Administration (NOAA) operates four different NOMADS Servers that host National Centers for Environmental Prediction (NCEP) Model Products. www. ncep.noaa.gov. ence the dispersion and deposition processes on a local scale. Therefore, in a second step, the global data can be adjusted to the near range around the release point by adapting the freely distributed mesoscale meteorological Weather Research Forecast model $(\mathrm{WRF})^{3}$ to the area under consideration. Currently, the WRF adaptation launcher is installed on a Linux platform (as WRF) with Java and can be invoked only manually; invoking within JRodos is foreseen for the near future.

The worldwide applicability of the system became available for all JRodos users in 2013. One of the first worldwide scenario applications was created for a demonstration of JRodos during a visit of a delegation from Saudi Arabia at the Karlsruher Institut für Technologie (KIT). The scenario introduced a fictive reactor situated in the Persian Gulf and a hypothetical release sequence. With respect to the meteorological conditions, reanalysis data in Grib2 format for September 2013 from the American NOMADS Server was used. Two screenshots from the JRodos calculation for this scenario are displayed in Figure 1 (total effective gamma dose rate, with OpenStreetMaps as the underlying map layer) and Figure 2 (wind field at $10 \mathrm{~m}$ height).

\section{Conclusions}

Modern decision support systems such as JRodos contain a multitude of features and tools that provide useful assistance when creating and running accident scenarios for case studies, demonstrations and training. Within the near future it will also become possible to apply JRodos for probabilistic risk assessments that require the analysis of a large number of weather sequences with otherwise identical other conditions.

\footnotetext{
${ }^{3}$ http://www.wrf-model.org/index.php.
} 


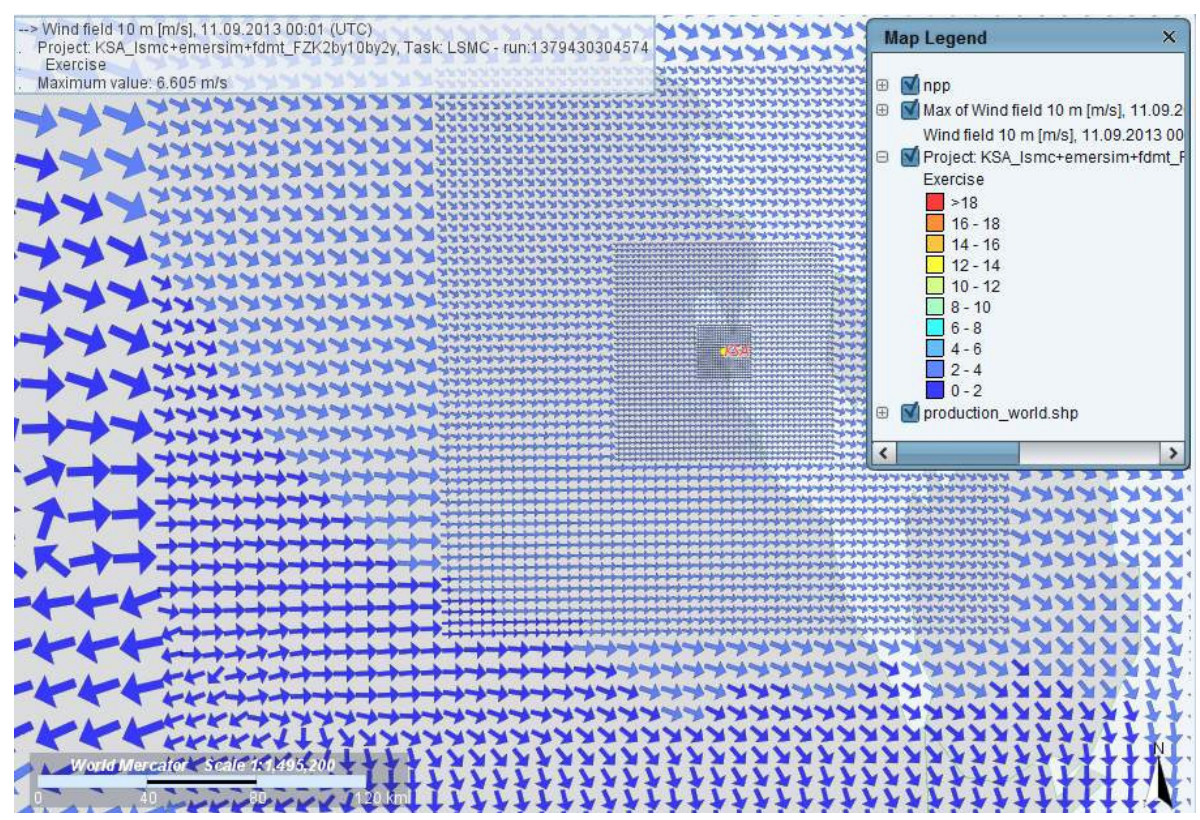

Figure 2. Wind field at $10 \mathrm{~m}$ height; NOMADS reanalysis data for 11.09.2013, for Saudi Arabia (JRodos screenshot).

Having the possibility of storing scenario components such as user input, required calculation data and application examples without much effort supports the distribution of complete scenarios among users and developers of the system, which can be beneficial for training purposes as well as for version control and documentation.

\section{References}

Chanin D.I. et al. (1990) MELCOR accident consequence code system (MACCS), User's Guide, NUREG/CR-4691, Albuquerque.
Gering F., Gerich B., Wirth E., Kirchner G. (2013) Potential Consequences of the Fukushima Accident for Off-Site Nuclear Emergency Management: A Case study for Germany, Radiat. Prot. Dosim., 155 (2), 146-154.

Ievdin I., Trybushnyi D., Zheleznyak M., Raskob W. (2010) RODOS reengineering: aims and implementation details. In: Enhancing nuclear and radiological emergency management and rehabilitation: Key results of the EURANOS European project (W. Raskob, M. Hugon, Eds.), Radioprotection 45 (5), Supplément 2010, S181-S189.

KfK, NRPB (1991) COSYMA - a new program package for accident consequence assessment, CEC Brussels, EUR 13028.

Cite this article as: C. Landman, W. Raskob, D. Trybushnyi, I. Ievdin. Creating and running worldwide accident scenarios with JRodos. Radioprotection 51(HS1), S27-S30 (2016). 